\title{
An emissions barrier stops spread of moisture driven emissions from buildings into the indoor air
}

\author{
Johan Mattsson $^{\mathrm{a}}$, Timo Lehtimaa ${ }^{\mathrm{b}}$, Lennart Larsson ${ }^{\mathrm{*}}$ \\ ${ }^{a}$ cTrap AB, Lund, Sweden, ${ }^{b}$ Sulin Oy, Helsinki, Finland, ${ }^{c}$ Lund University, Lund, Sweden
}

\begin{abstract}
A new type of emissions barrier was used in premises with indoor air complaints due to moisture driven emissions from the buildings in question. The emissions comprised chlorophenols/chloroanisoles and polycyclic aromatic hydrocarbons (PAH) from treated wood, and volatile organic compounds (VOC), mainly 2-ethylhexanol, from PVC flooring and the glue used to paste the flooring onto a concrete slab. Attaching the barrier at the surfaces from where the emissions were spread (floor, walls, ceiling) resulted in a fresh and odour-free indoor air. We conclude that using an emissions barrier in buildings made unhealthy by moisture is an efficient way of restoring a pleasant and healthy indoor air.
\end{abstract}

Peer-review under the responsibility of the organizing committee of the ICMB21.

Keywords: Emissions barrier; adsorbent; healthy buildings; restoration

\section{Introduction/Background}

Building moisture typically results in spread of chemical and biological emissions into the indoor air leading to illnesses and symptoms such as asthma, skin and eye irritation, fatigue etc. Drying is a necessary first step in remediation because it will stop further moisture-driven reactions with the building materials as well as (continued) mould growth. However, drying is not enough to secure a clean indoor air, since the numerous chemicals that have been formed from water - or moisture - acting on the materials will still remain in the building construction and over time inevitably be emitted into the indoor air resulting in an unsatisfactory indoor air quality (IAQ). The emissions may be e.g. VOC from paints, glue, insulation materials, chipboards, microorganisms, impregnation and plasticizer chemicals, or toxins from microorganisms such as mould. In the present study we applied a new type of emissions barrier [1,2] developed at Lund University Sweden to stop such emissions thereby improving the indoor air in buildings with IAQ complaints.

\section{Methods}

This study comprised three buildings with IAQ complaints due to emissions from the building construction. In short, the surfaces from where the emissions were spread (floor, ceiling, walls) were covered with a flexible emissions barrier to prevent them from reaching the indoor air. In the specific barrier used, the cTrap, an adsorption layer functions together with a hydrophilic polymer sheet making the adsorption virtually irreversible [1,2]. The flexible cTrap cloth was attached at the surfaces using an adhesive tape and/or a staple gun. Air concentrations of the emissions were measured both before and after the cTrap installations.

\section{Results}

We studied the living-room and a bedroom of a wooden summer house built in 1964 with a disturbing "summer cottage smell" which was attributed to chloroanisoles in the indoor air. The building had previously been treated with chlorophenolcontaining preservatives which were widely used in the 1960-70s; at moist conditions chlorophenols may be biomethylated to form chloroanisoles having an intense, characteristic mould-like odour. The ceiling, walls and floor in the bedroom (as well as the doorway between the bedroom and the living room) were covered with the cTrap cloth. Subsequently, air sampling for chlorophenols/chloroanisoles was carried out simultaneously in both rooms. Tetrachlorophenol, trichloroanisole, and pentachloroanisole were detected in the air of the living-room, but only tetrachlorophenol was found in the bedroom, and in an

* Corresponding author. +46725167879. lennart.larsson@med.lu.se

(C) The Author(s). This is an open access article distributed under the terms of the Creative Commons Attribution License (CC BY) 4.0 https:// creativecommons.org/licenses/by/4.0/, which permits unrestricted use, distribution and reproduction in any medium, provided the original author and source are credited. DOI: 10.14293/ICMB210042 


\section{ICMB21}

air concentration 93\% lower than in the living room. Also, the mouldy odour disappeared in the bedroom following the cTrap installation.

A building where a creosote-based tar layer had been attached onto the concrete slab as a moisture barrier was studied. The air concentrations of polycyclic aromatic hydrocarbons $(\mathrm{PAH})$ were $1726 \mathrm{ng} / \mathrm{m} 3$ air. There was a disturbing smell inside the building which persisted even after the tar had been removed. Then, the cTrap cloth was installed on about 75 percent of the wall surface. The smell disappeared and the PAH air concentration decreased to $139 \mathrm{ng} / \mathrm{m} 3$, thus corresponding to a reduction of $92 \%$.

A townhouse was studied where the tenants suffered from itching all over the body when staying at home, symptoms which disappeared when outside the building. A PVC flooring had been glued onto a concrete slab which had become moist through diffusion of water from the ground. The air concentration of 2-ethylhexanol, a compound which is ubiquitous in small concentrations in indoor air but found in increased concentrations e.g. following hydrolysis of glue and/or phthalates of PVC floorings, was $63 \mu \mathrm{g} / \mathrm{m} 3$ (directional measurement). The cTrap was attached onto the existing flooring, and the itchiness disappeared. 3 months after cTrap had been installed the air concentration was $1.5 \mu \mathrm{g} / \mathrm{m} 3$, a value which persisted in a follow-up study 6 years after the installation - and the residents still reported no symptoms.

Results are summarized in Table 1.

Table 1. Results of cTrap installations

\begin{tabular}{|l|l|l|}
\hline Emissions $\left(\mu \mathrm{g} / \mathrm{m}^{3}\right.$ air $)$ & Without cTrap & With cTrap \\
\hline Tetrachlorophenol & 0.14 & 0.01 \\
Chloroanisoles & 0.013 & n.d. \\
\hline PAH & 1.726 & 0.139 \\
\hline 2-ethylhexanol & 63 & 1.5 \\
\hline
\end{tabular}

\section{Discussion}

Staying in a moist building can cause health problems [3] e.g. in the respiratory tract due to moisture-driven emissions which are spread from the building construction into the indoor air. This study shows that such emissions can be effectively stopped by using an emissions barrier. Laboratory findings [2], as well as several years of experiences in buildings (data not shown), have revealed that by such measure the symptoms and/or unpleasant odour decrease or disappear completely. If any visible mould is observed it should first be removed by chemical or mechanical methods before applying the barrier to ascertain that no remaining mould products, or traces of the aggressive chemicals that might have been used, can escape into the indoor air. The specific device used in the current project, the cTrap (surface emissions trap), is air tight while at the same time allowing moisture to pass through with almost no resistance at all, and will thus not affect the moisture balance of the building. After the device has been applied on a floor, a surface layer, e.g. a laminate, parquet, or plastic flooring etc, is installed on top of the cTrap cloth. When attached on walls or ceiling the cloth is usually covered with a gypsum board which is then painted or decorated with a wall-paper. We conclude that use of an emissions barrier represents an effective, economic, and eco-friendly way of restoring a healthy indoor air in buildings affected by moisture.

\section{Conclusions}

An emissions barrier can be used to restore a fresh and healthy indoor air in buildings made unhealthy due to moisture damage.

\section{Acknowledgements}

Johnny Lorentzen is gratefully acknowledged for assisting in the chlorophenol/chloroanisole project.

\section{References}

[1] Markowicz, P, \& Larsson L, (2012). The surface emissions trap: A new approach in indoor air purification. J Microbiol Methods, 91, 290-294

[2] Markowicz, P, \& Larsson, L, (2015). Improving the indoor air quality by using a surface emissions trap. Atmos Environ, 106, $376-381$.

[3] Mendell, M, Mirer, A, Cheung, K, Tong, M, \& Douwes, J (2011). Respiratory and allergic health effects of dampness, mold, and dampness-related agents: a review of the epidemiologic evidence. Environ Health Perspect 119:6, 748-56. 